\title{
Distribution of three Pratylenchus species in chrysanthemum fields in the Kyushu-Okinawa region of southern J apan, with notes on their identification based on PCR-RFLP analysis.
}

\author{
Kenta Uesugi ${ }^{1}$, Hideaki Iwahori ${ }^{1}$ and Yasushi Tateishi ${ }^{1}$
}

\begin{abstract}
A survey to detect three Pratylenchus species, P. kumamotoensis, P. penetrans and P. pseudocoffeae, was conducted in chrysanthemum fields in the Kyushu-Okinawa region of southern Japan from 2005 to 2007. PCR-RFLP analysis was used for identification of extracted root lesion nematodes. The three species could be distinguished from one another by digestion patterns of Alu I, Hha I, Hinf I, or T aq I in a preliminary experiment and as a result Alu I and Hinf I were used for species identification in the field survey. A total of 86 soil samples were collected, and 38 samples (44\%) were infested with root lesion nematodes. Pratylenchus kumamotoensis was detected most frequently $(29 \%)$ and considered the most important root lesion nematode in this region. Detection rates of $P$. penetrans and $\mathrm{P}$. pseudocoffeae were $22 \%$ and $9 \%$, respectively. Pratylenchus penetrans was detected from Okinawa Prefecture for the first time. Nematol. Res. 39 (1), 17-22 (2009).

Key words : Pratylenchus kumamotoensis, Pratylenchus penetrans, Pratylenchus pseudocoffeae.
\end{abstract}

\section{INT RODUCTION}

Chrysanthemum (Chrysanthemum morifolium Ram.) is the most important flower used for ornamental and culinary purposes in Japan. Production area of chrysanthemum accounts for approximately onethird of the total cut flower production area, and onethird of the area is in the Kyushu-Okinawa region of southern Japan. Therefore, this region is an important target area for chrysanthemum pest control.

Root lesion nematode (Pratylenchus spp.) is one of the main soil pests of chrysanthemum. The nematode causes severe root lesions, resulting in crop failure when susceptible cultivars are grown successively (Kobayashi, 1995; Mizukubo et al., 2007). Five species of the genus Pratylenchus have been detected from chrysanthemum fields in Japan, namely P. penetrans (Cobb), P. pseudocoffeae Mizukubo, P. kumamotoensis M izukubo et al, P. fallax Seinhorst, and Pratylenchus sp. 2 of Gotoh (1974) (Gotoh, 1974; Mizukubo, 1992a; Mizukubo, 1992b; Mizukubo et al., 2007). Pratylenchus penetrans has been recognized as causing damage to chrysanthemum since the 1960's (Kobayashi, 1968). This species is polyphagous and has been widely detected from the Hokkaido region to the Kyushu-Okinawa region in Japan (Gotoh, 1974). In contrast to $P$. penetrans, the species $P$. pseudocoffeae and $P$. kumamotoensis were described relatively recently, with

${ }^{1}$ National A gricultural Research Center for Kyushu Okinawa Region, 2421, Suya, K oshi, Kumamoto 861-1192, Japan. few known hosts and with few detection records in the Kyushu-Okinawa region (Mizukubo, 1992a; Mizukubo et al., 2007; Iwahori et al., 2008). These three species may be involved with chrysanthemum damage in the KyushuOkinawa region, where their distributions overlap. Records of the other two species, P. fallax and Pratylenchus sp. 2 of Gotoh (1974), were not based on conclusive data (Mizukubo, 1992b) and are not considered here.

The recent discovery of $P$. pseudocoffeae and $P$. kumamotoensis boosted the need for a revision of species causing damage to chrysanthemum in the KyushuOkinawa region. For the development of an appropriate control strategy, it is important to elucidate the prevalence of the three species based on an updated extensive field survey. However, available data (Gotoh, 1974; M izukubo, 1992a; Mizukubo et al., 2007) are obsolete or based on small-scale field surveys. Consequently, the status of the three species as chrysanthemum pests in the region still remains unclear.

In the present study, we surveyed chrysanthemum fields in the Kyushu-Okinawa region to elucidate the current prevalence of the three lesion nematodes. We used PCR-RFLP analysis for species identification and showed its effectiveness in detecting root lesion nematodes related to chrysanthemum in a field survey.

\section{MATERIALS AND METHODS}

Soil sample collection:

Soil samples were collected from 86 chrysanthemum 
fields in Kyushu (Kyushu Island) and Okinawa (Okinawa Island) from 2005 to 2007 (Fig. 1); six open fields in Fukuoka Prefecture, two open fields and four greenhouses in Saga Prefecture, one open field and nine greenhouses in Nagasaki Prefecture, three greenhouses in Oita Prefecture, seven open fields and 13 greenhouses in Kumamoto Prefecture, one greenhouse in M iyazaki Prefecture, eight open fields and 16 greenhouses in Kagoshima Prefecture, and 14 open fields and two greenhouses in Okinawa Prefecture. The 86 fields included the two fields from which the Shibushi population and the Koshi population of P. kumamotoensis used in Mikuzubo et al. (2007) were obtained. Sampling seasons and cultural conditions of examined fields, e.g. chrysanthemum cultivars and growth stages, were not standardized. Soil samples were collected from the rhizosphere of chrysanthemum at more than five points of each field with a $25 \mathrm{~cm}$-long shovel and then approximately $2 \mathrm{~kg}$ soil in total was mixed well in a plastic bag.
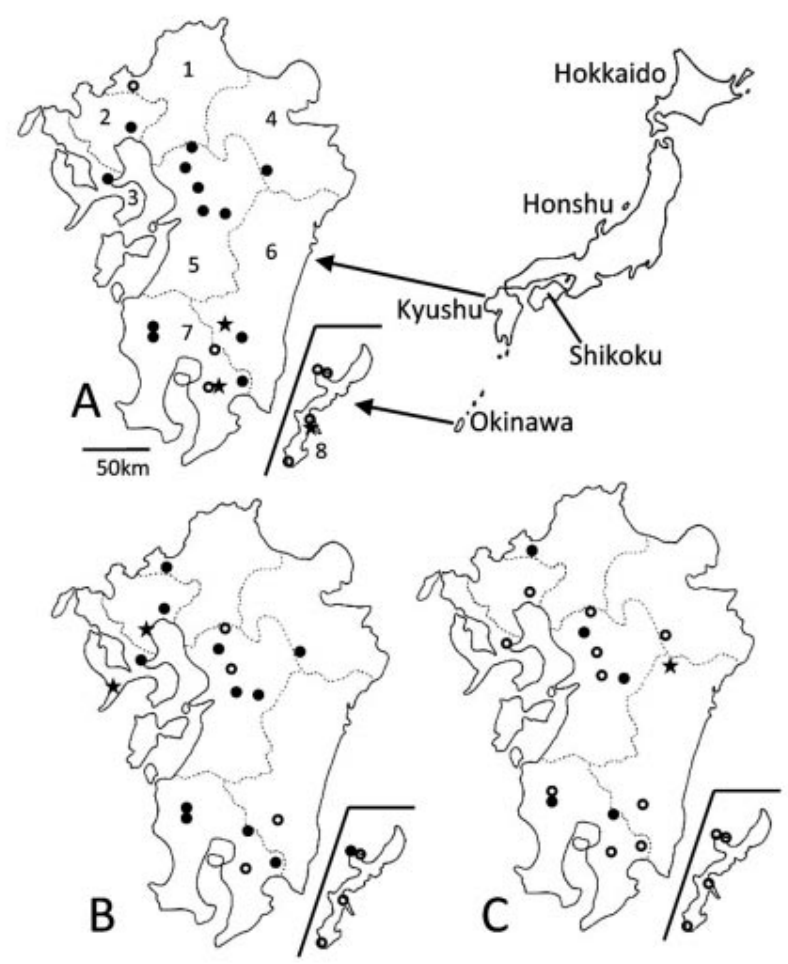

Fig. 1. Geographic position of the regions in Japan and chrysanthemum fields in the Kyushu-Okinawa region with Pratylenchus species detected; (A) P. kumamotoensis, (B) P. penetrans, (C) P. pseudocoffeae. One to ten neighboring fields examined are represented by one circle. White circles indicate the species was not detected. Black circles indicate the species was detected at least in one field. Stars indicate approximate positions of infested fields reported in Gotoh (1972), Mizukubo (1992), Mizukubo et al. (2007) and Iwahori et al. (2008). Numbers in A indicate prefectures: 1, Fukuoka; 2, Saga; 3, Nagasaki; 4, Oita; 5, Kumamoto; 6, Miyazaki; 7, Kagoshima; 8, Okinawa.
Nematode extraction and counts of Pratylenchus spp.:

Nematodes were extracted from two $20 \mathrm{~g}$ soil samples using the Baermann funnel technique for three days at room temperature. The numbers of root lesion nematodes were counted with a microscope under $\times 60$ or $\times 150$ magnification. The average of the two counts was used as a datum.

Identification to the species level:

Ten to 13 females and/or juveniles of root lesion nematodes were randomly selected and identified to the species level by PCR-RFLP analysis in each sample. If the number of root lesion nematodes was fewer than ten in the first extraction, an additional extraction was conducted. If multiple extractions still resulted in fewer than ten nematodes, then all nematodes extracted were identified (five samples). In one sample, 23 individuals (females and juveniles) were also identified to confirm mixed infestation by two species of Pratylenchus.

Because RFLP profiles of $P$. kumamotoensis and $P$. pseudocoffeae were unknown, we first examined the profiles of these two species as follows. The Kanoya (Kagoshima Prefecture) population of $\mathrm{P}$. kumamotoensis and $Y$ oron (Kagoshima Prefecture) population of $P$. pseudocoffeae were used. The Motobu (Okinawa Prefecture) population of $\mathrm{P}$. penetrans was also used for comparison. All populations were propagated from a single gravid female and kept on alfalfa callus or on monoxenic chrysanthemum culture. The populations were identified by morphometrics of adult females with the aid of sequencing analysis of D2/D3 expansion of ribosomal DNA (rDNA) for P. pseudocoffeae (736 of 739 compared base pairs were identical with the Florida population (A F 170444) presented in Duncan et al. (1999)). DNA was extracted from a single nematode by a filter paper method (Iwahori et al., 2000). A $n$ autoclaved $1 \times 1 \mathrm{~mm}$-filter paper with a crushed nematode was placed in $5 \mu$ buffer containing $0.1 \mu$ P Proteinase $\mathrm{K}, 10 \mathrm{mM} \mathrm{Tr}$ ris- $\mathrm{HCl}$ and $1 \%$ Nonidet $\mathrm{P}-40$, incubated at $65^{\circ} \mathrm{C}$ for $1 \mathrm{hr}$ followed by $95^{\circ} \mathrm{C}$ for 10 min and stored at $-80^{\circ} \mathrm{C}$. A filter paper without a nematode was used as the negative control. The internal transcribed (ITS) regions of rDNA were amplified using the primer set of Ferris et al. (1993), 5'-CGT A A C A A G GT A GCT GTA G-3' and 5'-TCC TCC GCT AAA TGA TAT G-3'. PCR was performed in a $25 \mu$ reaction mix containing 5 $\mu$ of DNA template, $0.4 \mu \mathrm{M}$ primers and $0.5 \mathrm{U}$ of TaKaRa Ex Taq ${ }^{\circledR}$ (TaKaRa). PCR amplification conditions were as follows: 35 cycles with denaturation at $94^{\circ} \mathrm{C}$ for 1 min, annealing at $48^{\circ} \mathrm{C}$ for $1 \mathrm{~min}$, and extension at $72^{\circ} \mathrm{C}$ for 2 min with a final extension at $72^{\circ} \mathrm{C}$ for $5 \mathrm{~min}$. Four endonucleases, A lu I, Hha I, Hinf I, and T aq I, were 
used for digestion with reference to Orui and Mizukubo (1999a). Four $\mu \mathrm{l}$ of the PCR products were digested with $0.5 \mathrm{U}$ of endonuclease in a total volume of $5 \mu \mathrm{l}$ at $37^{\circ} \mathrm{C}$ (A lu I, Hha I and Hinf I) or $65^{\circ} \mathrm{C}$ (T aq I) overnight. The PCR products and the RFLP patterns were analyzed by $2.0 \%$ agarose gel electrophoresis in TBE buffer.

In the identification of field samples, Alu I and Hinf I digestion patterns were examined for each extracted nematode. These two endonucleases were used as standard endonucleases for PCR-RFLP analysis in previous field surveys of various crops (Orui and Mizukubo, 1999b; Orui and Mizukubo, 1999c; Iwahori et al., 2008). A nalysis was conducted as described above except for partial usage of T aKaRa T aq ${ }^{\circledR}$ (T aKaRa) and GoT aq ${ }^{\circledR}$ Greem Master M ix (Promega) in PCR.

\section{RESULTS}

PCR-RFLP analysis:

The three species were clearly distinguished by PCRRFLP analysis (Fig. 2). The size of PCR product was ca. $800 \mathrm{bp}$ in P. kumamotoensis and P. penetrans and ca. 1000 $b p$ in $P$. pseudocoffeae. The four endonucleases cut all PCR products of the three species. The PCR-RFLP profile of $P$. penetrans was identical to that in Orui and Mizukubo (1999a). T wo faint bands, ca. 450 bp and ca. 340 bp in addition to the ca. 800 bp band, were observed in the Hinf I digestion pattern of P. kumamotoensis, indicating the partial digestion of the PCR product.
Field survey:

Root lesion nematodes were detected in 38 of 86 fields examined (44\%) (T able 1, Fig. 1). A total of 393 root lesion nematodes were identified, of which 230 were $P$. kumamotoensis, 96 were $P$. penetrans, and 61 were $P$. pseudocoffeae. Six root lesion nematodes collected in three fields of Kagoshima Prefecture were not identified because their RFLP profiles were different from any known profiles (data not shown). No variation was observed in the RFLP profiles of $P$. penetrans and $P$. pseudocoffeae. On the other hand, the approximately $210 \mathrm{bp}$ band in Alu I digestion patterns of $\mathrm{P}$. kumamotoensis was often indistinct and produced variation even among individuals from the same soil sample (Fig. 3).

The detection rate of lesion nematodes was $53 \%$ in Kyushu, whereas it was as low as $6 \%$ in Okinawa (T able 1). The detection rate in open fields was $67 \%$ in Kyushu (n $=24)$ and $0 \%$ in Okinawa $(n=14)$. The detection rate in greenhouses was $46 \%$ in Kyushu $(n=46)$ and $50 \%$ in Okinawa $(n=2)$.

The detection rates of $\mathrm{P}$. kumamotoensis $(29 \%)$ and $\mathrm{P}$. penetrans $(22 \%)$ were higher than P. pseudocoffeae $(9 \%)$ (Table 1). T wo or three species were detected together in approximately one-third of the overall Pratylenchuspositive fields (T able 1). The species combinations in these fields were $P$. kumamotoensis $+P$. penetrans in 9 fields, $P$. kumamotoensis $+P$. pseudocoffeae in 1 field, $P$. penetrans $+P$. pseudocoffeae in 2 fields, and all three species in 1

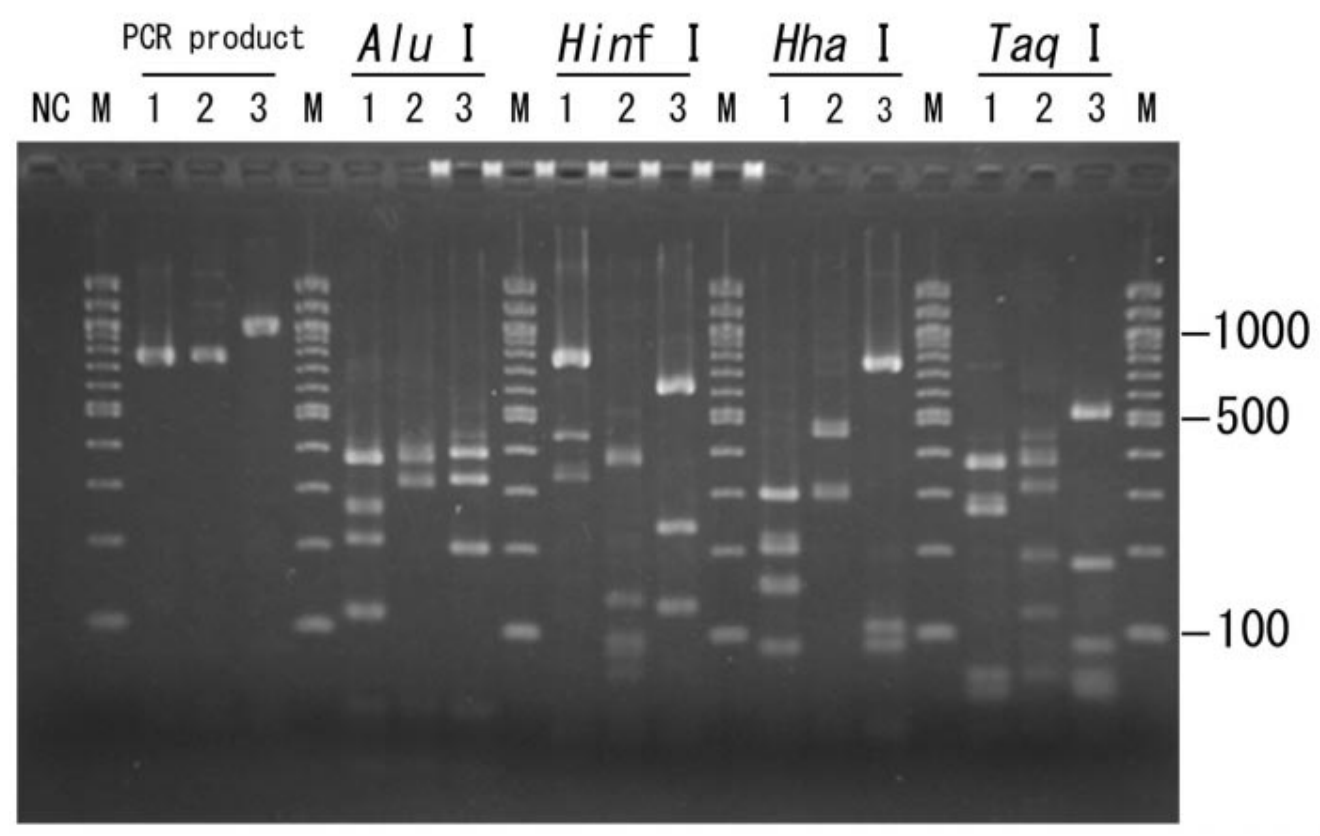

Fig. 2. $P C R$ products of $P$. kumamotoensis, $P$. penetrans and $P$. pseudocoffeae and their RFLP patterns after digestion by each of the four endonucleases, Alu I, Hha I, Hinf I and T aq I. Lane 1, P. kumamotoensis; lane 2, P. penetrans; lane 3, P. pseudocoffeae; M, 100 bp ladder marker; NC, negative control. 
Table 1. Numbers of chrysanthemum fields in the Kyusyu-Okinawa region with Pratylenchus detected.

\begin{tabular}{lcccccc}
\hline \multirow{2}{*}{ Region } & Prefecture & $\begin{array}{c}\text { Number of } \\
\text { fields }\end{array}$ & Pratylenchus & \multicolumn{4}{c}{ Species $^{1}$} \\
\cline { 5 - 7 } & examined & positive & P. kumamotoensis & P. penetrans & P. pseudocoffeae \\
\hline Kyushu & Fukuoka & 6 & $2(0)^{2}$ & 0 & 1 & 1 \\
& Saga & 6 & $1(1)$ & 1 & 1 & 0 \\
& Nagasaki & 10 & $1(1)$ & 1 & 1 & 0 \\
& Oita & 3 & $3(1)$ & 3 & 1 & 0 \\
& Kumamoto & 20 & $13(3)$ & 8 & 5 & 4 \\
& Miyazaki & 1 & $1(0)$ & 1 & 0 & 0 \\
& Kagoshima & 24 & $16(8)$ & 11 & 9 & 3 \\
Okinawa & Okinawa & 16 & $1(0)$ & 0 & 1 & 0 \\
\hline \multirow{2}{*}{ Total } & & 86 & $38(14)$ & 25 & 19 & 8 \\
& & $(100 \%)$ & $(44 \%(16 \%)$ & $(29 \%)$ & $(22 \%)$ & $(9 \%)$ \\
\hline
\end{tabular}

${ }^{1}$ Unidentified species are omitted.

${ }^{2}$ Numbers in parenthesis indicate fields infested with more than one species.

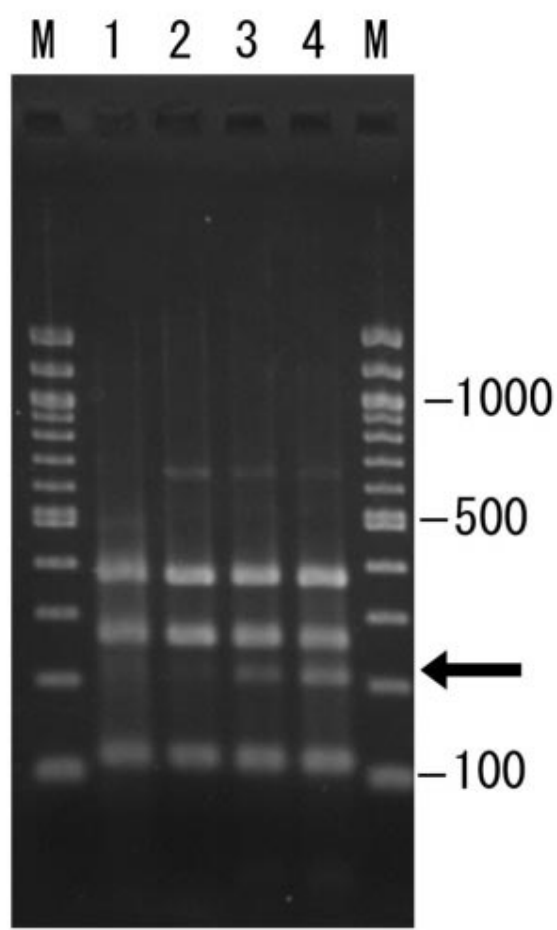

Fig. 3. Variation in Alu I RFLP pattern in P. kumamotoensis. Lanes 1-4, four individuals collected from the same chrysanthemum field in Saga Prefecture; M, 100 bp ladder marker; arrow, 210 bp band.

field (unidentified species were not considered). Nematode densities were higher than 100 nematodes in $20 \mathrm{~g}$ soil in 14 fields (T able 2). A II three species were dominant in more than one of these highly infested fields.

\section{DISCUSSION}

The distribution of lesion nematodes in the present field survey was clearly different to the previous distribution map of $P$. kumamotoensis, P. penetrans and P. pseudo- coffeae in Kyushu-Okinawa region chrysanthemum fields. They previously have been sporadically recorded in this region; P. penetrans from Saga and Nagasaki Prefecture (Gotoh, 1974), P. pseudocoffeae from Miyazaki Prefecture (Mizukubo, 1992a) and P. kumamotoensis from Kumamoto, Miyazaki, Kagoshima, and Okinawa Prefecture (M izukubo et al., 2007; I wahori et al., 2008). Our study clearly showed that the three species are widely distributed in the region and that Pratylenchus-positive chrysanthemum fields are frequently infested with more than one species. The detection rate of root lesion nematodes was high even in greenhouse cultivation, where the appropriate use of nematicides is expected. Considering the high detection rates and wide distributions, $P$. kumamotoensis and $P$. penetrans should be regarded as the main targets for nematode control in this region. Pratylenchus pseudocoffeae was also present in Kumamoto and Kagoshima Prefectures, although its detection rate was relatively low all over the region.

It should be noted that $P$. kumamotoensis was most frequently detected in the Kyushu region. This species had not been recorded in chrysanthemum fields before Mizukubo et al. (2007). Its distribution in natural habitats is also poorly understood, with a record from a habitat with Artemisia princeps growing in Kumamoto Prefecture (Mizukubo et al., 2007). Our study could not determine whether P. kumamotoensis has been rapidly expanding in distribution or if its wide distribution had been merely overlooked in the previous studies. However, the latter case seems to be less likely since $P$. kumamotoensis reproduces at an extremely high rate in various chrysanthemum cultivars (Uesugi, unpublished data), and field population densities were often extremely high in our survey. The rapid dispersal of the species due to artificial transfer 
Table 2. Population density distribution and dominant species in 38 chrysanthemum fields infested with Pratylenchus spp.

\begin{tabular}{cccccc}
\hline \multirow{2}{*}{$\begin{array}{c}\text { Population } \\
\text { density }\end{array}$} & \multirow{2}{*}{$\begin{array}{c}\text { Number of } \\
\text { fields }\end{array}$} & \multicolumn{5}{c}{ Dominant species $^{2}$} \\
\cline { 3 - 6 } & & P. kumamotoensis & P. penetrans & P. pseudocoffeae & Undetermined \\
\hline$<10$ & 12 & 3 & 2 & 2 & 5 \\
$10-100$ & 12 & 9 & 1 & 1 & 1 \\
$100-1000$ & 11 & 4 & 3 & 2 & 2 \\
$>1000$ & 3 & 3 & 0 & 0 & 0 \\
\hline
\end{tabular}

${ }^{1}$ Number of root lesion nematodes extracted from $20 \mathrm{~g}$ soil by Baermann funnel technique $(72 \mathrm{hr})$

${ }^{2}$ The species which occupied $70 \%$ or more of the individuals identified. When no such species occured or the number identified was less than 10, the field was classified " Undetermined" .

of infested nursery stock is thus more likely.

The distribution of $P$. penetrans was once considered to be sporadic in the Kyushu region (Gotoh, 1974). However, the number of detections has been increasing since the 1980's (Mizukubo, 1992b), and now our results show that $P$. penetrans is no longer an uncommon species in chrysanthemum fields in the region. Pratylenchus penetrans causes severe damage to edible burdock and Japanese radish (Sano and Nakazono, 1987; Shiraishi, 1995). Chrysanthemum fields could be potential sources of secondary dispersal of $P$. penetrans to these crop fields.

Pratylenchus penetrans was recorded from Okinawa Prefecture, a subtropical region, for the first time. Pratylenchus kumamotoensis was also recorded in this prefecture recently (Iwahori et al., 2008). The detection rate of root lesion nematodes in Okinawa was low, it is thus important to prevent further spread of these species from, e.g., infested nursery stock (Gotoh, 1974).

Despite the agricultural importance, mixed infestation has not been sufficiently illustrated in morphology-based surveys (Gotoh, 1974). M eanwhile, our study showed that approximately one-third of Pratylenchus-positive fields were infested with more than one species. The rate of mixed infestation was probably underestimated due to the small number of identified nematodes, less than 13 individuals in each sample with one exception. The rate is rather high compared to those observed in tobacco and some other crop fields of central and northern Japan (Orui and Mizukubo, 1999b; Orui and Mizukubo, 1999c). It is obvious that a nematode control strategy for mixed infestation is required in the Kyushu region, especially when a cultural control is applied. Further understanding of the biology of P. kumamotoensis and P. pseudocoffeae is essential for developing appropriate control strategies for these species.

Nematode damage to chrysanthemum in examined fields was not measured in the present study. Kobayashi (1995) reported a decrease in shoot height with root lesion nematode (species unspecified) at densities below 100 nematodes in $20 \mathrm{~g}$ soil by Baermann funnel extraction at early growth stage. Nematode densities in one-third of Pratylenchus-positive fields were higher than 100 in $20 \mathrm{~g}$ soil in our data. Nematode densities in these fields may remain high and above the control threshold at planting of the following crop, because a fallow period is generally short in year-round chrysanthemum cultivation. The differences in pathogenicity and control thresholds among the three species should be examined.

We used PCR-RFLP analysis for nematode identification in the field survey. PCR-RFLP analysis is suitable for practical use because it is a less time-consuming procedure than morphological methods or other molecular methods such as sequencing analysis. PCR-RFLP profiles of ITS regions of rDNA have been accumulated for identification of the major Japanese species of Pratylenchus, including $P$. penetrans (Orui, 1996; Orui and Mizukubo, 1999a). Although the PCR-RFLP profile of a European population of $P$. pseudocoffeae was presented by $W$ aeyengerge et al. (2000), the results of this study were not comparable because the authors used primer sets different from those in Orui and Mizukubo (1999a). Our study followed the protocol of Orui and Mizukubo (1999a) including the primer set selection of Ferris et al. (1993) in order to compare the PCR-RFLP profiles with those of the other Japanese congeneric species. The three species were clearly distinguished from one another and from other species examined in Orui and Mizukubo (1999a) by the four endonucleases. Practical identification in the field survey was successfully conducted by RFLP analysis using only Alu I and Hinf I, though a small variation was observed in the digestion pattern of Alu I in P. kumamotoensis. Orui and Mizukubo $(1999 b, c)$ also reported the effectiveness of these two endonucleases in the field surveys of other Pratylenchus species. As far as hitherto analyzed species are concerned, all Japanese Pratylenchus species can be identified by the digestion pattern of these two endonucle 
ases.

\section{ACKNOWLEDGEMENTS}

We are grateful for arrangement of sample collection to $S$. Kawasaki, H. T origoe and A. Hayashi of Kagoshima prefectural government, A. Ohshiro, T. Zakimi and K. Zaha of Okinawa prefectural government, $\mathrm{T}$. F uruie, $\mathrm{K}$. Obara and $\mathrm{E}$. Kaneko of Kumamoto prefectural government, K. T eramoto of JA Kamoto, S. Okazaki, Y. T oida and $T$. Suehiro of Oita prefectural government, $Y$. Kagino of Nagasaki prefectural government, and $Y$. Miyazaki of Saga prefectural government.

\section{LITERATURE CITED}

Duncan, L. W., Inserra, W. K., Dunn, D., M ustika, I., Frisse, L. M., Mendes, M. L., Morris, K. and Kaplan, D.T. (1999) Molecular and morphological analysis of isolates of Pratylenchus coffeae and closely related species. Nematropica 29, 61-80.

Ferris, V. R., Ferris, J. M . and Faghihi, J. (1993) Variation in spacer ribosomal DNA in some cyst-forming species of plant parasitic nematodes. Fundamental and A pplied Nematology 16, 177-184.

Gotoh, A . (1974) Geographic distribution of Pratylenchus spp. (Nematoda: T ylenchida) in Japan. Bulletin of the Kyushu Agricultural Experiment Station 17, 139-224. (in Japanese with English summary)

Iwahori, H., Kanzaki, N. and Futai K. (2000) A simple, polymerase chain reaction-restriction fragment length polymorphism-aided diagnosis method for pine wilt disease. Forest Pathology 30, 157-164.

Iwahori, H., Uesugi, K. and T ateishi, Y. (2008) Plant parasitic nematode fauna in upland field crops, fruit trees and weeds in Okinawa Prefecture, Japan. Proceedings of the A ssociation for Plant Protection of Kyushu 54, 132-137. (in Japanese with English summary)

Kobayashi, Y. (1968) T he damage of chrysanthemum plants caused by Pratylenchus penetrans. Bulletin of the Shizuoka A gricultural Experiment Station 13, 114123. (in Japanese with English summary)

Kobayashi, Y. (1995) The analysis of the damages of Chrysanthemum morifolium Ramat. caused by nematodes and their control measures. Technical Bulletin No.19, Shizuoka A gricultural Experiment Station, Japan, 77pp. (in Japanese with English summary)

Mizukubo, T. (1992a) Pratylenchus pseudocoffeae n. sp.
(Nematoda: Pratylenchidae) from composite plants in Japan. A pplied Entomology and Zoology 27, 437-444.

Mizukubo, T. (1992b) The classification, morphology and distribution of the genus Pratylenchus in Japan. In: Progress in Nematology. (Nakazono, K. ed.), Japanese Nematological Society, T sukuba, Japan, 40-47. (in Japanese)

Mizukubo, T., Sugimura, K. and Uesugi, K. (2007) A new species of the genus Pratylenchus from chrysanthemum in Kyusyu, western Japan (Nematoda: Pratylenchidae). Japanese Journal of Nematology 37, 63-74.

Orui, Y. (1996) Discrimination of the main Pratylenchus species (Nematoda: Pratylenchidae) in Japan by PCRRFLP analysis. A pplied Entomology and Zoology 31, 505-514.

Orui, Y . and Mizukubo, T . (1999a) Discrimination of seven Pratylenchus species (Nematoda: Pratylenchidae) in Japan by PCR-RFLP analysis. A pplied Entomology and Zoology 34, 205-211.

Orui, Y . and Mizukubo, T. (1999b) Geographical distribution of Pratylenchus species in tobacco fields in eastern Japan. Japanese Journal of A pplied Entomology and Zoology 43, 75-79. (in Japanese with English summary)

Orui, Y . and Mizukubo, T . (1999c) Species composition of Pratylenchus (Nematoda: Tylenchida) in crop fields of central Japan, along with an observation of the geographic border lying between Pratylenchus coffeae and its closely allied species. Japanese Journal of Nematology 29, 1-6. (in Japanese with English summary)

Sano, Z. and Nakazono, K. (1987) First record of Pratylenchus penetrans causing severe damage to edible burdock in central Kyushu, Japan. Japanese Journal of Nematology 17, 57-58. (in Japanese)

Shiraishi, T . (1995) Plant-parasitic nematodes in vegetable fields in Oita Prefecture and damage to Japanese radish caused by root-lesion nematode, $P$. penetrans. Proceedings of the Association for Plant Protection of Kyushu 41, 93-95. (in Japanese)

W aeyenberge, L., Ryss, A., M oens, M., Pinochet, J. and Vrain, T. C. (2000) Molecular characterization of 18 Pratylenchus species using rDNA restriction fragment length polymorphism. Nematology 2, 135-142.

Received October 28, 2008 